\title{
A Markovian Model for the Analysis of Age of Information in IoT Networks
}

\author{
Qamar Abbas, Syed Ali Hassan, Haris Pervaiz and Qiang Ni
}

\begin{abstract}
Age of Information (AoI) is a critical metric in status update systems as these systems require the fresh updates. This paper investigates the uplink of an Internet-of-thing (IoT) network where $L$ nodes transmit their information packets to a base station. The effects of the arrival rate of packets at the nodes, the number of nodes in the system, and queue length of each node have been studied by devising a discrete time Markov chain (MC) model. This model helps in predicting the values of AoI and probability of packet drops in such systems. The notion of first-in first-out is used for queuing, which transmits the oldest packet first, resulting in decreasing the overall AoI of the system. The results show that AoI increases with the increase in queue length, number of nodes and arrival rate and we quantify the aforementioned metrics using the MC model.The results found using the MC model are also validated using extensive simulations.
\end{abstract}

Index Terms-Internet-of-thing, age of information, Markov chain, arrival rate, queuing theory.

\section{INTRODUCTION}

W Ith the explosive growth of Internet-of-thing (IoT) systems, real-time status updates have become a crucial and ubiquitous form of communication. Examples of such status update systems include smart agriculture, smart traffic control systems, smart homes and smart cities, etc. To quantify the information freshness about these remote systems, age of information (AoI) has been recently introduced [1]. AoI is defined as the time elapsed since the generation of the latest successfully received update about the source system. The notion of AoI is different from throughput and delay because system utilization can be maximized by allowing the nodes to send the arrived packets as soon as possible [2]. However, this may result in backlogging of the communication system. Similarly, the delay can be reduced by decreasing the number of updates, but this can result in obsolete packets because of the lack of fresh updates.

The characterization of information freshness in also paramount in wide range of applications. AoI has been studied so far as a concept, performance metric and a tool [3]. In real-time monitoring of IoT systems, AoI is crucial to be considered as the stale information can degrade the system performance in such systems. For example, in [4], AoI is

Q. Abbas and S. A. Hassan are with the School of Electrical Engineering \& Computer Science (SEECS), National University of Sciences \& Technology (NUST), Pakistan. (e-mail:\{qabbas.phdee17seecs, ali.hassan\}@ seecs.edu.pk).

Haris Pervaiz and Qiang $\mathrm{Ni}$ are with the Department of Computing and Communications Lancaster university, UK (e-mail: h.b.pervaiz@lancaster.ac.uk; q.ni@lancaster.ac.uk).

This work is supported in parts from the Higher Education Commission (HEC) of Pakistan and Global Challenges Research Fund (GCRF) for project UTS1000XS14. critical in agricultural monitoring as the latest information is needed for precision agriculture applications and hence precision agriculture along with IoT can be used to enhance productivity of agriculture crops by monitoring soil properties, moisture level, meteorological behavior, etc [5]. Similarly, [6] models the problem of real-time scheduling based on AoI in wireless ad hoc networks by using a scheduling policy to improve the AoI without sacrificing the feasibility optimality. A simplified method to evaluate AoI based on stochastic hybrid system using a finite-state Markov chain is formulated for a multiple source network sharing a single server [7]. The authors proposed closed-form AoI expressions for simple queue models and the results are compared for different queuing systems. [8] uses deadlines to drop the outdated packets in a downlink IoT network updating multiple nodes. AoI is evaluated using a fixed and random deadlines and the advantages of respective deadlines in different deadline regimes is shown.

Although recent studies focus on minimizing the AoI, however, the probability of packet drops in such systems is also important to discuss. This letter provides a performance analysis of an uplink IoT system with the help of Markov chains where the number of nodes and the queue length in each node is used to quantify both the AoI and the probability of packet drops. Specifically, we assume a first-in first-out (FIFO) queue model where each information packet carries a distinct information such as in a multi-sensory scenario and each information packet is critical to reach the receiver with a minimum AoI. A related example is [9], which considers FIFO queuing which stores all newly-arrived status packets and gives higher priority to the older status packets whereas the newly-arrived packets are preempt in the queue till the transmission of all older packets. However, when the buffer is full, the queuing policy still blocks the latest update until the transmission of older packets. This leads to the growth of overall age unlike our model which discards the oldest information from head of the queue instead to tail dropping. Similarly, [10] compares FIFO and M/G/1/1 queuing for diverse class of source content and it is shown that for a constant service rate and regular arrivals from a source, FIFO policy outperforms other policies for a large interval of high priority stream generation rate.

In this work, we use a discrete time BER/G/1/Q queuing model where the arrivals occur at a time instant with probability $\lambda$ following a Bernoulli distribution, and a queue length $Q$ as analyzed in [11] and compute the probability of packet drops and AoI of the network using the state transition matrix of Markov chain. The main contributions of this letter are 
outlined below.

- We model the AoI and probability of packet drops using a discrete time Markov chain (MC) model and study various attributes of network performance by analyzing the state probability distribution.

- We quantify the effects of number of nodes, queue length and arrival rate on AoI and packet drops using the proposed analytical model.

\section{SySTEM MOdeL}

Consider the uplink of a wireless network consisting of a base-station (BS) and $L$ sensor nodes denoted by the set $\mathbb{L}=\left\{l_{1}, \ldots, l_{L}\right\}$, having a queue length, $Q$, each where each node extracts real-time information from an environmental source. Each node transmits information to the BS in the form of packets, where the inter-arrival times are independent and the size of all packets is considered the same. We consider a BER/G/1/Q queuing model where the arrival process follows a Bernoulli distribution with parameter $\lambda$ and the processing time at each node does not exceed one time slot.

When a packet arrives at the queue of a sensor, it waits in the queue where each sensor has a queue length of $Q$. The waiting time of $i^{t h}$ node increases as $L$ increases because the total transmission time depends on the number of nodes. Similarly, the waiting time increases as $Q$ increases hence, with the increase in $L$ and $Q$, the information freshness of each node is also compromised. We assume that every packet is carrying unique information and when a node has $Q$ packets in its queue and another packets arrives, the oldest packet is discarded from the queue to ensure the information freshness. The discarded packets due to the queue overloading are counted as packet drops. We now model the AoI and the probability of packet drops, $P_{\text {drops }}$, using a Markov chain, which is described in the next section.

\section{PRoposed MARKov MODEL}

At a certain time $n$, the state of the system can be described as the number of packets waiting for transmission in the queues of each sensor and the decision metric for transmission at the BS. Hence, the state of system at time $n$, represented as $X(n)$ is given as

$$
X(n)=\left\{\mathbb{S}_{1}(n), \mathbb{S}_{2}(n), . ., \mathbb{S}_{L}(n), \mathbb{D}_{1}(n), \mathbb{D}_{2}(n), . ., \mathbb{D}_{L}(n)\right\}
$$

where $\mathbb{S}_{i}(n), i \in\{1,2, \ldots L\}$ shows the number of packets waiting in the queue of the $i^{\text {th }}$ sensor at time $n$ and its value is given as a $Q$-tuple indicator function, i.e.,

$\mathbb{S}_{i}(n)= \begin{cases}0 & \text { if the node has no packets in queue } \\ 1 & \text { if the node has one packet in queue } \\ \vdots & \\ \mathrm{Q} & \text { if the node has } \mathrm{Q} \text { (maximum) packets in queue. }\end{cases}$

Similarly the BS decision for the $i^{t h}$ node is also a binary indicator function such that for $i^{\text {th }}$ node and at time $n$,

$$
\mathbb{D}_{i}(n)= \begin{cases}0 & \text { if the node is not transmitting } \\ 1 & \text { if the node is transmitting. }\end{cases}
$$

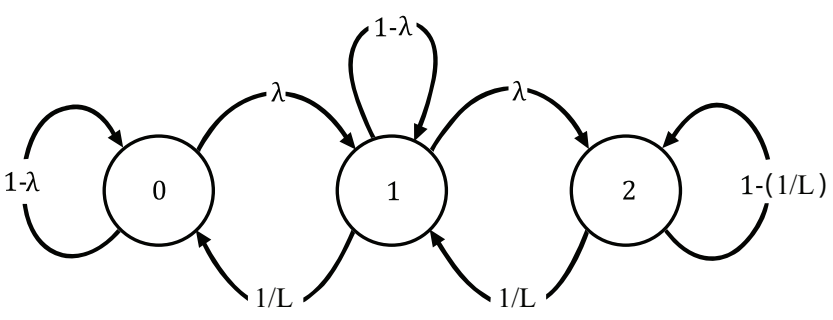

Fig. 1: The state transition diagram of a single node for $Q=2, L=2$

Note that as only one node can transmit at one time, therefore $\mathbb{D}_{i}(n)=1$ implies $\sum_{\forall n} \mathbb{D}_{f}(n)=0$, i.e., all other nodes are not transmitting.

The next state of the system only depends on the current state and the arrival probability, $\lambda$, on all nodes satisfying the Markovian property. We now divert our attention on the state transition of the $i^{t h}$ node. The transition of the $i^{t h}$ node to state $\mathbb{S}_{i}(n+1)$ given that the current state is $\mathbb{S}_{i}(n)$ depends on $\mathbb{D}_{i}(n)$ and $\lambda$. For instance, if the current state of $i^{t h}$ node, i.e., $\mathbb{S}_{i}(n)=1$ and $\mathbb{D}_{i}(n)=1$, the next state of the node will be 0 with a unit probability as the only packet in its queue is transmitted. Similarly, if $\mathbb{S}_{i}(n)=1$ and $\mathbb{D}_{i}(n)=0$, the next state of the node will be 2 with probability $\lambda$ (packet arrives) or $\mathbb{S}_{i}(n+1)=1$ with probability $1-\lambda$ as shown in Fig. 1 . When a node is in $Q$ state, the oldest packet will be replaced by the new arrived packet. This implies that, when the current state of a node is $Q$ and an arrival occurs, the oldest packet will be dropped until any packet is transmitted to change the current state to any state having a value less than $Q$.

The next state of the $i^{t h}$ node given that the node is in state $S_{i}(n)$ at a time $n$ is given as,

$$
\mathbb{S}_{i}(n+1)= \begin{cases}\mathbb{S}_{i}(n) & \text { if } \mathbb{D}_{i}(n)=0 \text { and no arrival } \\ \mathbb{S}_{i}(n)+1 & \text { if } \mathbb{D}_{i}(n)=0 \text { and arrival } \\ \mathbb{S}_{i}(n)-1 & \text { if } \mathbb{D}_{i}(n)=1 .\end{cases}
$$

The total number of states for the Markov chain $X$ given $L$ and $Q$ is denoted as $L_{S}$ and is given as

$$
L_{S}=L \times(Q+1)^{L} .
$$

Suppose there are two nodes, i.e., $L=2$ and queue length of each sensor is 2 i.e., $Q=2$. The BS allocates a time slot for transmission to the two sensors randomly. The probability of transmission in this case will be $1 / 2$ whereas the total number of transition states will be 18 according to (5). Suppose both sensors have a single packet waiting in their queue, and the first node gets a time slot for transmission. The current state of the system will be $X(n)=1110$, where the first two digits indicate that both sensors have one packet in their queues and the last two digits show the decision of the BS, respectively. If the BS allows transmission to the first sensor, the next possible states will be $X(n+1)=0110$ or $X(n+1)=0101$, given that there is no arrival on both nodes. In this example, the state of the first node becomes 0 as the waiting packet is transmitted and there is no arrival on the first node in this slot. The second 
node is not transmitting, therefore, there is a probability of arrival $\lambda$ on the second node. The state of the second node will be 2 if arrival occurs or the node will remain in state 1 otherwise. If there is an arrival on the second node, the next possible states are $X(n+2)=0210$ or $X(n+2)=0201$ and this process continues.

Considering the above discussions, let the first $L$ digits of the system state of $X(n)$ are represented by the vector $\mathbf{a}_{n}$ such that $\mathbf{a}_{n} \in \mathbf{Z}^{+(1 \times L)}$ where $\mathbf{Z}^{+}$is the set of positive integers including zero and the last $L$ digits of the system state are represented by the vector $\mathbf{b}_{n}$. The purpose is to find the transition probability of the system from the state $X(n)=\left\{\mathbf{a}_{n} \mathbf{b}_{n}\right\}$ to the state $X(n+1)=\left\{\mathbf{a}_{n+1} \mathbf{b}_{n+1}\right\}$, where we need the values of $\alpha, \beta$ and $\gamma$, which are found using Algorithm 1. In the algorithm, $\gamma$ counts the number of nodes having a full queue, whereas $\alpha$ denotes the number of nodes having arrival at a time instant $n$. Moreover, $\beta$ represents the number of nodes that remain in the same state and have less than $Q$ packets in their queues.

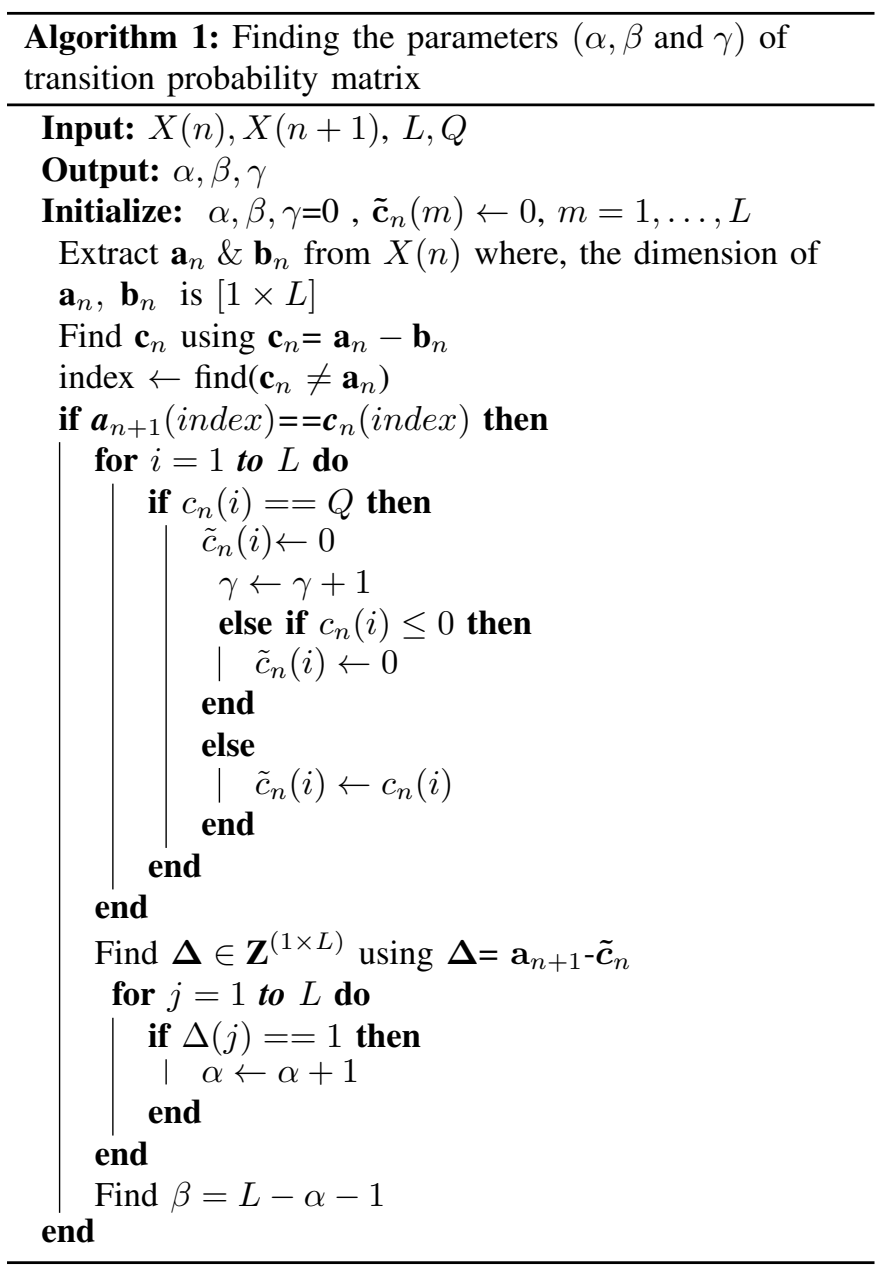

For any pair of states $X(n)$ and $X(n+1)$, the following propositions are applied to calculate the transition probability from $X(n)$ to $X(n+1)$ :

Proposition 1. For the states $X(n)$ and $X(n+1)$, when any element of the vector $\boldsymbol{\Delta}$ does not belong to $\{0,1\}$, $\mathbb{P}_{(X(n), X(n+1)) \mid(\mathbb{D}(n))}=0$.
Proposition 2. For the states $X(n)$ and $X(n+1)$, when all elements of the vector $\boldsymbol{\Delta}$ belong to $\{0,1\}$, the transition probability is given as

$$
\mathbb{P}_{(X(n), X(n+1)) \mid(\mathbb{D}(n))}=\frac{1}{L}\left(\lambda^{\alpha}(1-\lambda)^{\beta-\gamma}\right) .
$$

The transitions to and from all transient states are incorporated in a transition probability matrix, $\mathbf{P}$, for which a single entry is given by Proposition 1 or 2 . Note that the matrix $\mathbf{P}$ is sparse owing to the fact that many transitions are prohibited given the state space of the system. After the formation of stochastic matrix, using the property of $\mathrm{MC}$, let $\mathbf{v}$ be the eigenvector of $\mathbf{P}$ corresponding to the eigenvalue $\chi$, then,

$$
(\mathbf{P}-\chi \mathbf{I}) \mathbf{v}=\mathbf{0} .
$$

The eigenvector $\mathbf{v}$ after normalization provides the state probability distribution of the system $\phi$, which is further used to calculate the AoI as described in the next section.

\section{Age of Information Model}

Consider a packet, $p$, is generated at a node at time $u(n)$, then the age of that packet can be written as

$$
\psi_{p}(n)=n-u(n)
$$

where $n$ is the current time instant. AoI of a packet is incremented by 1 at next time slot if the packet is not transmitted while it becomes zero if it is transmitted. According to the above discussion, AoI in the next slot of any packet which is oldest packet in a node is written as

$$
\psi_{p}(n+1)= \begin{cases}0 & \text { if } \mathbb{D}_{i}(n+1)=1 \\ \psi_{p}(n)+1 & \text { if } \mathbb{D}_{i}(n+1)=0\end{cases}
$$

following the well known sawtooth pattern introduced in [1].

As the length of the vector $\mathbb{S}_{i}(n)$ shows the number of packets currently waiting in the queue of the $i^{t h}$ node, we can find the AoI of the $i^{\text {th }}$ node by summing the ages of all packets currently waiting in its queue. Let the length of vector $\mathbb{S}_{i}(n)$ at time $n$ be $\kappa$ and $\psi_{p}(n)$ be the AoI of the $p^{t h}$ packet in $i^{\text {th }}$ node then,

$$
\operatorname{AoI}_{i}(n)=\sum_{p=1}^{\kappa} \psi_{p}(n)
$$

If the state of the $i^{t h}$ node at time $n$ is $\mathbb{S}_{i}(n)$, then the AoI at time $n+1$ will be summation of AoI in the current time slot, i.e., $\operatorname{AoI}_{i}(n)$, and the state $\mathbb{S}_{i}(n)$ with probability $1-\lambda$ when $\mathbb{D}_{i}(n+1)=0$. Similarly, when $\mathbb{D}_{i}(n+1)=0$, the AoI at time $n+1$ will be summation of AoI in the current time slot, i.e., $\operatorname{AoI}_{i}(n)$, and the state $\mathbb{S}_{i}(n)+1$ with probability $\lambda$. If $\mathbb{D}_{i}(n+1)=1$, the AoI at time $n+1$ will be $\operatorname{AoI}_{i}(n)-\mathbb{S}_{i}(n)$ with unit probability. The AoI for $i^{t h}$ node at BS at time $n+1$ can be expressed as,

$\operatorname{AoI}_{i}(n+1)=\left\{\begin{array}{l}\operatorname{AoI}_{i}(n)+\mathbb{S}_{i}(n) \quad \text { if } D_{i}(n+1)=0 \text { and no arrival } \\ \operatorname{AoI}_{i}(n)+\mathbb{S}_{i}(n)+1 \quad \text { if } D_{i}(n+1)=0 \text { and arrival } \\ \operatorname{AoI}_{i}(n)-\mathbb{S}_{i}(n) \quad \text { if } D_{i}(n+1)=1\end{array}\right.$ 


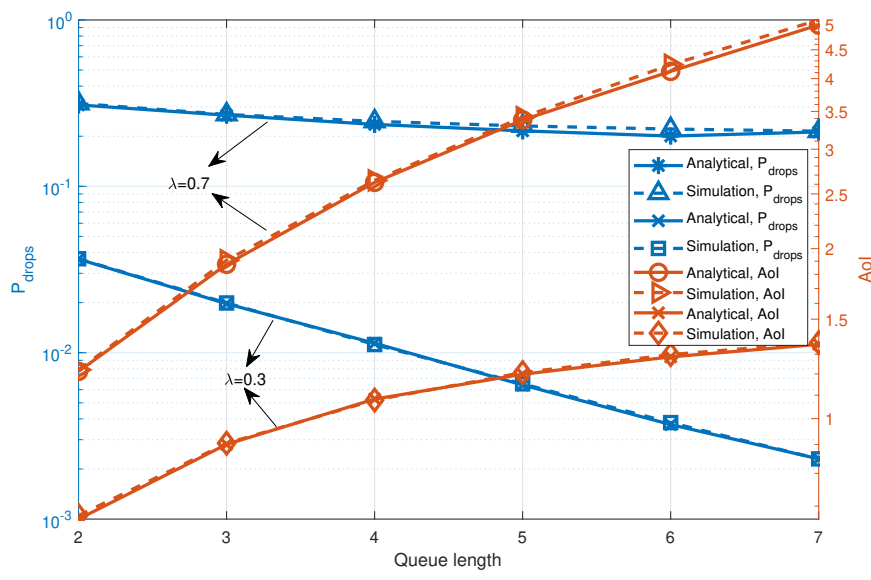

Fig. 2: AoI and $P_{\text {drops }}$ trade-off for queue length, $Q$, and $L=3$

TABLE I: Mean Absolute Error

\begin{tabular}{|l|l|l|l|l|}
\hline $\boldsymbol{\lambda}$ & $\mathbf{L}=\mathbf{3 , Q = 2}$ & $\mathbf{L}=\mathbf{3 , Q}=\mathbf{Q}$ & $\mathbf{L}=\mathbf{4 , Q = 2}$ & $\mathbf{L = 5 , Q = 2}$ \\
\hline $\mathbf{0 . 1}$ & $0.034 \%$ & $0.040 \%$ & $0.020 \%$ & $0.013 \%$ \\
\hline $\mathbf{0 . 3}$ & $0.025 \%$ & $0.038 \%$ & $0.017 \%$ & $0.010 \%$ \\
\hline $\mathbf{0 . 5}$ & $0.021 \%$ & $0.037 \%$ & $0.016 \%$ & $0.007 \%$ \\
\hline $\mathbf{0 . 7}$ & $0.017 \%$ & $0.023 \%$ & $0.015 \%$ & $0.006 \%$ \\
\hline $\mathbf{0 . 9}$ & $0.013 \%$ & $0.015 \%$ & $0.012 \%$ & $0.004 \%$ \\
\hline
\end{tabular}

As per our assumption above, the BS schedules transmission of each node unbiasedly and the arrival $\lambda$ on each node is kept same. Therefore, the AoI at each node will also be same. When we find the AoI at any random node, we can also find AoI of the entire system, $\overline{\mathrm{AoI}}$, at $n+1$ using (11) as

$$
\overline{\operatorname{AoI}}(n+1)=\sum_{i=1}^{L} A o I_{i}(n+1) .
$$

Similarly, the probability of packet drops $\left(P_{d r o p s}\right)$ on each node will also be equal as it is a function of $\lambda$ and the state of a node. $P_{d r o p s}$ at the $i^{\text {th }}$ node can be found using the state distribution vector of the system $\phi$. The probability of packet drops on the $i^{t h}$ node can be given as,

$$
P_{\text {drops }}=\frac{(L-1) \lambda}{L} \times \sum_{j=y}^{L_{s}} \phi(j)
$$

where, $y=\left(Q\left(\frac{L_{s}}{L}+1\right)+1\right) /(Q+1)$.

Note that the AoI derived in (11) and (12) does not include transmission impairments where it is assumed that each transmitted packet reaches the BS with unit success probability. However, for a more realistic receiver model, we consider a Rayleigh fading channel between a node and the $\mathrm{BS}$, where the outage probability at a given SNR threshold, $\Gamma$, is given as, $P_{\text {out }}=1-e^{-\Gamma\left(P_{t} / \sigma^{2}\right)}$, where, $P_{t}$ is the transmit power of a node and $\sigma^{2}$ is noise power spectral density. Therefore, the effective AoI with fading can be found as, $A o I_{\text {eff }}=A o I /\left(1-P_{\text {out }}\right)$. It can be seen that as the outage event of a packet increases, the effective AoI also increases.

\section{PERFormance EVAlUATION AND DISCUSSIONS}

This section discusses the performance of the network in terms of AoI and probability of packet drops for different

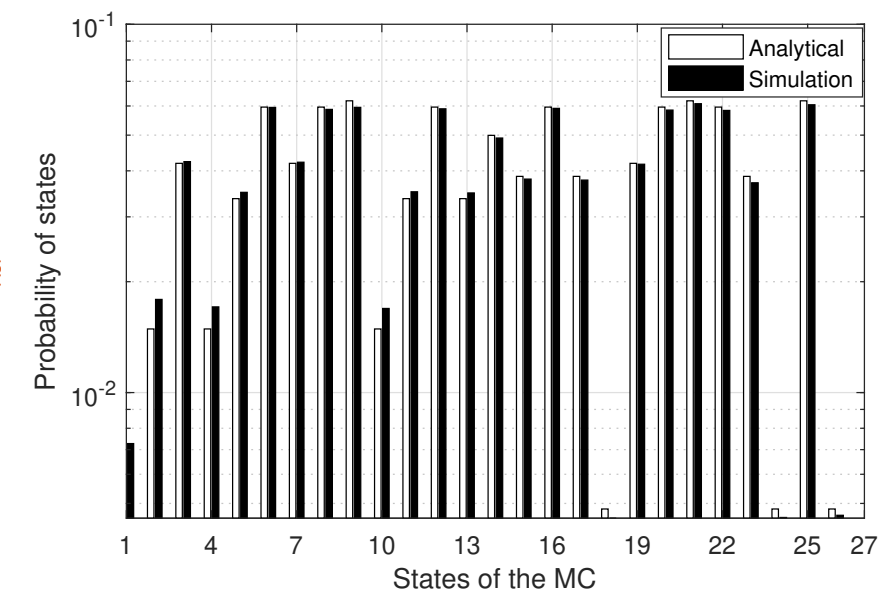

Fig. 3: The state probability distribution of the system for $L=3, Q=3$ and $\lambda=0.7$

queue lengths and the arrival rates on each node. The results are found analytically using Markov chain and compared with the simulation results.

In Fig. 3, the state probability distribution is shown for $L=3, Q=3$ and $\lambda=0.5$ using the proposed MC model. The horizontal axis shows the states of the system which has 27 length for the given combination of $L$ and $Q$ calculated using Eq. (5) and vertical axis shows the probability of all states. The hollow bars show the probability distribution using the MC model while the filled bars are numerical simulation results. It can be seen that the proposed analytical model has a close agreement with simulations, thereby validating the $\mathrm{MC}$ model. As the arrival rate is 0.5 , therefore, the graph is not skewed on either side. If $\lambda>0.5$, the graph shifts to right side because the probability of states representing larger number of packets in the queue increases and vice versa. The mean absolute error between the analytical and simulation results is computed in Table I for various values of $L, Q$ and $\lambda$ and it can be seen that the proposed model is valid for a variety of parameter combinations.

Fig. 2 shows the trade-off between AoI and probability of packet drops. The y-axis on left side of the graph shows the probability of packet drops, while the y-axis on the right side denotes AoI without transmission errors. It can be seen that an increase in the queue length increases the AoI while it decreases the probability of packet drops. The results are shown for arrival rates of 0.3 and 0.7 and also validated using simulation results. For a given $L$, the AoI increases with an increase in $Q$ because with an increase in the queue length, the stale information packets will reside in the queue for longer times instead of dropping with queue overloading in smaller queue lengths. Similarly, for a constant queue length, when $L$ increases, the AoI also increases because with an increase in $L$, the transmission probability of a node decreases. An increase in $Q$, decreases the packet drops because there will be more space for the packets in the queue. The result also depicts that the packet drops decrease with an increase in queue length for a given number of nodes. 


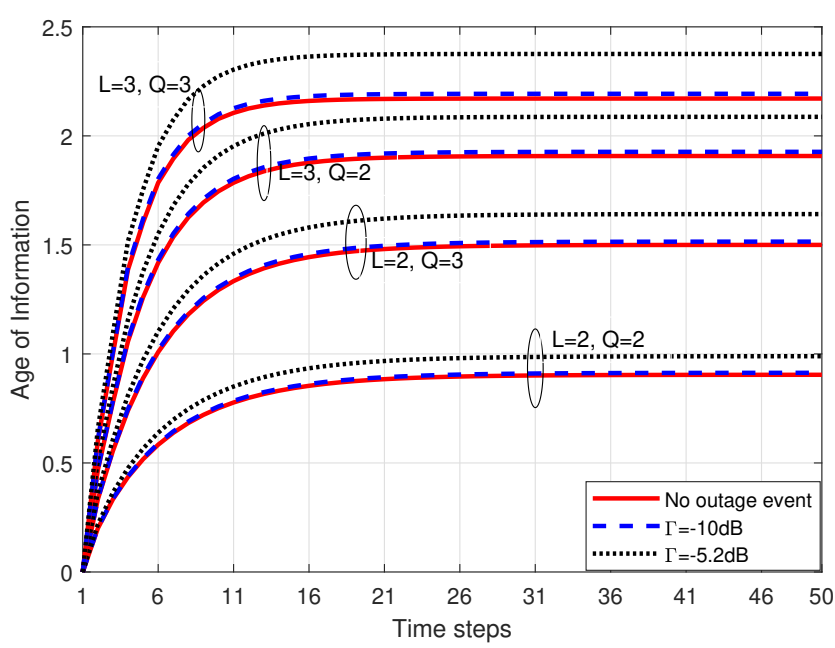

Fig. 4: Evolution of AoI with number of time steps for $\lambda=0.5$

In Fig. 4, the evolution of AoI with the time steps is shown for different number of nodes and different queue lengths for $\lambda=0.5$. For each $L$ and $Q$ pair, the results are compared for different SNR thresholds i.e., no outage, $\Gamma=-10 d B$ and $\Gamma=-5.2 d B$ for operating SNR of $0 \mathrm{~dB}$. It can be seen that initially, with the increase in the number of steps, the AoI increases however, it becomes constant after a steady state is reached. The result also indicates that AoI increases with increment in queue length and number of nodes. Furthermore, with the increase in $\Gamma$, the outage probability increases which results in increasing the AoI. It can also be noticed that when the number of nodes and/or queue length increases, the number of states increases therefore, the steady state is attained after large time steps.

Similarly, Fig. 5 shows the evolution of AoI with the number of time steps for different arrival rates. The results are simulated for $L=3$ and $Q=3$ for different arrivals and outage values at $0 \mathrm{~dB}$ SNR. The results indicate that AoI increases with the arrival rate because when the arrival rate increases, the number of packets in the queue becomes large. Likewise, when $\Gamma$ is increased, the outage probability is also increased incrementing the AoI. It can also be seen that a larger arrival rate also results in obtaining the steady state earlier as compared to lower values of arrival rate.

\section{CONCLUSION}

This letter investigated the effects of the arrival rate of packets, queue length, and the number of nodes in an uplink IoT system on the AoI and probability of packet drops using a MC model. The accuracy of the approach is evaluated using mean absolute error between the probability distribution of the proposed MC and simulation results. The results showed that the probability state distribution, AoI and probability of packet drops are function of arrival rate, queue length and number of nodes in the system. Further investigations to the current work include incorporating different queuing models in the study such as last-in first-out (LIFO) where the service/processing time of a packet may have a longer duration than a single time

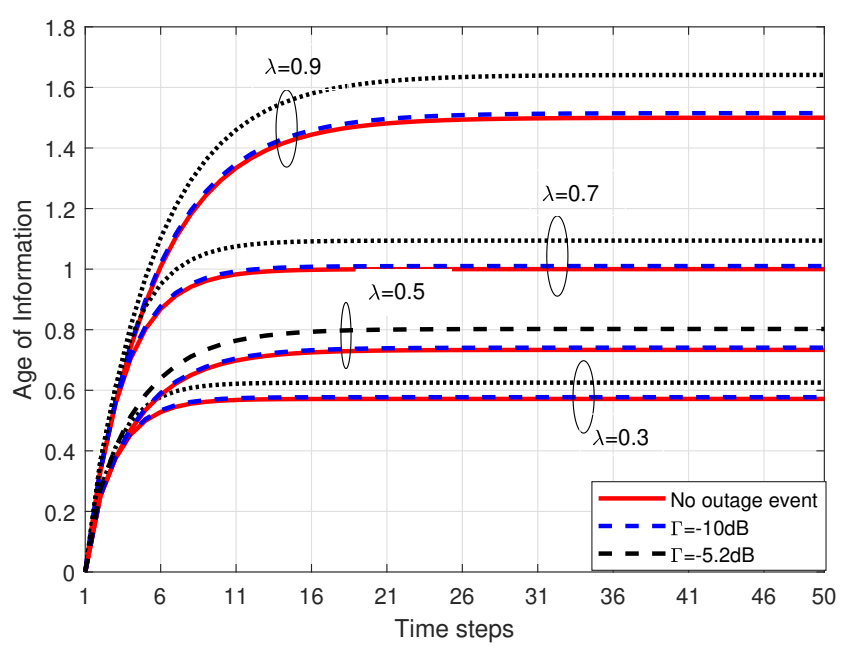

Fig. 5: Evolution of AoI with number of time steps for $L=3, Q=3$

slot. Similarly, the optimization of queue length to achieve a desired AoI under an outage probability constraint is another future direction to this work.

\section{REFERENCES}

[1] S. Kaul, M. Gruteser, V. Rai, and J. Kenney, "Minimizing age of information in vehicular networks," in 2011 8th Annual IEEE Communications Society Conference on Sensor, Mesh and Ad Hoc Communications and Networks, 2011, pp. 350-358.

[2] S. Kaul, R. Yates, and M. Gruteser, "Real-time status: How often should one update?" in 2012 Proceedings IEEE INFOCOM, 2012, pp. 27312735.

[3] A. Kosta, N. Pappas, and V. Angelakis, "Age of information: A new concept, metric, and tool," Foundations and Trends in Networking, vol. 12, no. 3, pp. 162-259, 2017.

[4] Q. Abbas, S. Zeb, S. A. Hassan, R. Mumtaz, and S. A. R. Zaidi, "Joint optimization of age of information and energy efficiency in IoT networks," in 2020 IEEE 91st Veh. Technol. Conf. (VTC2020-Spring), 2020 , pp. $1-5$.

[5] Shafi, Uferah and Mumtaz, Rafia and García-Nieto, José and Hassan, Syed Ali and Zaidi, Syed Ali Raza and Iqbal, Naveed, "Precision agriculture techniques and practices: From considerations to applications," Sensors, vol. 19, no. 17, p. 3796, 2019.

[6] N. Lu, B. Ji, and B. Li, "Age-based scheduling: Improving data freshness for wireless real-time traffic," in Proceedings of the Eighteenth ACM International Symposium on Mobile Ad Hoc Networking and Computing, 2018, pp. 191-200.

[7] R. D. Yates and S. K. Kaul, "The age of information: Real-time status updating by multiple sources," IEEE Trans. Inform. Theory, vol. 65, no. 3, pp. 1807-1827, 2018.

[8] J. Li, Y. Zhou, and H. Chen, "Age of information for multicast transmission with fixed and random deadlines in iot systems," IEEE Internet of Things Journal, 2020.

[9] Zheng, Xi and Zhou, Sheng and Jiang, Zhiyuan and Niu, Zhisheng, "Closed-form analysis of non-linear age of information in status updates with an energy harvesting transmitter," IEEE Trans. on Wireless Comm., vol. 18, no. 8, pp. 4129-4142, 2019.

[10] Najm, Elie and Nasser, Rajai and Telatar, Emre, "Content based status updates," IEEE Trans. on Info. Theory, vol. 66, no. 6, pp. 3846-3863, 2019.

[11] V. Tripathi, R. Talak, and E. Modiano, "Age optimal information gathering and dissemination on graphs," in IEEE INFOCOM 2019-IEEE Conference on Computer Communications. IEEE, 2019, pp. 24222430. 